\title{
Borio, Claudio u. a. (Hrsg.): Promoting Global Monetary and Financial Stability. The Bank for International Settlements after Bretton Woods, 1973-2020, 302 S., Cambridge UP, Cambridge 2020.
}

\section{Matthias Kemmerer}

Angenommen: 29. April 2021 / Online publiziert: 22. Mai 2021

(C) Der/die Autor(en) 2021

Die Bank für Internationalen Zahlungsausgleich (BIZ) diente bei ihrer Gründung 1930 dem Zweck, die deutschen Reparationszahlungen nach dem Ersten Weltkrieg neu zu regeln. Mittlerweile ist die in Basel ansässige ,Bank der Zentralbanken “ eine internationale Finanzorganisation, die die globale Währungs- und Finanzstabilität fördert. Anlässlich ihres 90. Jubiläums hat die BIZ einige namhafte Autoren beauftragt, ihre jüngere Entwicklung nach dem Ende des Währungssystems von Bretton Woods 1973 nachzuzeichnen. Auf die luzide Einleitung der Herausgeber folgen fünf sich thematisch ergänzende Kapitel sowie ein knapper Erfahrungsbericht eines früheren Insiders. Der Band verbindet so Geschichts- und Rechtswissenschaften, Makroökonomie und Internationale Politische Ökonomie. Obwohl zeitlich überlappend, vermeiden die Texte Redundanzen. Souverän führen sie die bis zum Jahr 1973 reichende Gesamtdarstellung von Gianni Toniolo (2005) und die Spezialstudie von Charles Goodhart (2011) zum Basler Bankenausschuss fort. Zwar wurde die dreißigjährige Archivsperrfrist zum Teil aufgehoben. Wie bei Goodhart waren gleichwohl nur ausgewählte vertrauliche Dokumente zugänglich, was den Neuigkeitswert bisweilen schmälert.

Harold James beschreibt die Beziehung der BIZ zur Europäischen Währungsunion. Die europäischen Zentralbankgouverneure koordinierten ihre Politik zwischen den 1960er und frühen 1990er Jahren nicht in Brüssel, sondern bei der BIZ in Basel, die ihnen als Sekretariat diente. Der neutrale Schweizer Tagungsort symbolisierte die Haltung der Zentralbanker, die sich als von der Politik unabhängige, aber selbst politisch machtlose Technokraten verstanden. James' Narrativ ähnelt dem, das er virtuos bereits in seinem 2012 erschienenen Grundlagenwerk zur Europäischen Währungsunion entfaltet hat. Inwiefern die BIZ das europäische Währungsexperiment konkret beeinflusste, bleibt im vorliegenden Beitrag allerdings etwas offen.

Dies mag auch daran liegen, dass die BIZ keine geschlossene Institution war. Sie besaß, so Catherine Schenk, stets eine wandlungsfähige governance: Neben dem Verwaltungsrat, der heute aus Mitgliedszentralbanken mit ständigen und alternieren-

M. Kemmerer ( $\square)$

Goethe-Universität Frankfurt am Main, Frankfurt a. M., Deutschland

E-Mail: matthias.kemmerer@stud.uni-frankfurt.de 
den Sitzen besteht, bestimmte lange der Club der weltweit wichtigsten Notenbanken - die ,Zehnergruppe " - die inhaltliche Arbeit, die in den Ausschüssen geleistet wurde. Als dritter Machtfaktor emanzipierte sich seit den 1990er Jahren das Management der BIZ in Basel von den Mitgliedsbanken. Vor allem der Generaldirektor und die Chefökonomen wurden eigenständiger. Schenk beschreibt eindrücklich die Globalisierungswehen einer traditionell eurozentrischen Institution: Die ,Zehnergruppe“ wollte die intime Club-Atmosphäre erhalten, in der man Meinungen vertraulich austauschen konnte. Dagegen drängten außereuropäische Zentralbanken auf größeren Einfluss. Die BIZ musste inklusiver werden, um ihre Legitimität und Autorität zu wahren. Erst die Politik verschob seit der Weltfinanzkrise 2008/9 das Gewicht: Die Staats- und Regierungschefs der G20 schufen das Financial Stability Board (FSB) als neues, breiteres Forum, um Fragen der globalen Finanzstabilität zu erörtern; die ,Zehnergruppe " wurde aufgelöst. Das bei der BIZ in Basel ansässige FSB hat seither den Einfluss des BIZ-Managements weiter vergrößert.

Chris Brummer betont, dass die BIZ über Jahrzehnte ein modernes soft law schuf, indem in ihrem Einflussbereich unverbindliche Absichtserklärungen entstanden und Ideen entwickelt wurden, die das globale Währungs- und Finanzsystem stabilisieren sollten. Leider verpasst es Brummer, diese Prozesse näher zu erläutern, da er kaum Primärquellen auswertet. Er zeigt aber, wie die BIZ und ihre Ausschüsse Ideen für Institutionen wie den Internationalen Währungsfonds (IWF) lieferten, die wiederum für die Umsetzung ins politische hard law zuständig waren.

Andrew Baker rekonstruiert erstmals auf Archivbasis, wie das BIZ-Management Ende der 1990er Jahre begann, das bei Zentralbankern und Aufsehern vorherrschende prozyklische Denken zu hinterfragen. Das Management trat vielmehr für einen antizyklischen Ansatz ein: Risiken für das Finanzsystem seien nicht in Rezessionen am größten, sondern in Boomphasen. Das BIZ-Management befürwortete öffentlich eine makroprudenzielle Finanzaufsicht, die das gesamte Finanzsystem - und nicht einzelne Finanzinstitute (mikroprudenziell) - in den Mittelpunkt stellte. Seit der Finanzkrise ist diese neue Sichtweise als internationaler Standard anerkannt. Die ständige Reflexion des globalen Finanzsystems (,measured contrarianism“) ist so zum Wesenskern der BIZ geworden.

Barry Eichengreen wertet die Jahresberichte der BIZ aus, um aus den dortigen Einlassungen des Mitarbeiterstabs die Ursprünge, der' BIZ-Sichtweise heraus zu präparieren. Obschon Eichengreen gelegentlich Aktenfunde mit einbezieht, wirkt sein Vorgehen teils additiv. Aufschlussreich ist, dass bereits der frühere Generaldirektor Alexandre Lamfalussy vor der lateinamerikanischen Schuldenkrise der 1980er Jahre makroprudenzielles Denken vorantrieb. Eichengreen sieht zurecht in den weiterhin nationalen Notenbankmandaten das größte Hindernis, um internationalen Konsens herzustellen.

Der Band endet mit dem kurzen Erfahrungsbericht des früheren Präsidenten der New York Federal Reserve, William C. Dudley, der nach den voranstehenden Kapiteln aber kaum Neues bietet.

Wirtschaftshistoriker werden dieses Buch mit Gewinn lesen. Über die vielfachen Meinungsverschiedenheiten der ,Zehnergruppe ' in den Ausschüssen hätte man zwar gerne mehr erfahren. Auch der 1971 eingesetzte Ständige Euromarktausschuss wird nur gestreift. Deutlich wird aber, dass in erster Linie US-amerikanische Ver- 
treter allzu lange am prozyklischen und mikroprudenziellen Denken festhielten, auf dessen Defizite die BIZ-Mitarbeiter vor der Finanzkrise mehrfach hinwiesen. Globalisierungshistorisch und mikropolitisch veranschaulicht der Band, wie internationale (Finanz-)Organisationen sich von ihren Ursprungsträgern emanzipieren und über ihr Personal zu eigenen Spielern der Weltwirtschaft werden.

Funding Open Access funding enabled and organized by Projekt DEAL.

Open Access Dieser Artikel wird unter der Creative Commons Namensnennung 4.0 International Lizenz veröffentlicht, welche die Nutzung, Vervielfältigung, Bearbeitung, Verbreitung und Wiedergabe in jeglichem Medium und Format erlaubt, sofern Sie den/die ursprünglichen Autor(en) und die Quelle ordnungsgemäß nennen, einen Link zur Creative Commons Lizenz beifügen und angeben, ob Änderungen vorgenommen wurden.

Die in diesem Artikel enthaltenen Bilder und sonstiges Drittmaterial unterliegen ebenfalls der genannten Creative Commons Lizenz, sofern sich aus der Abbildungslegende nichts anderes ergibt. Sofern das betreffende Material nicht unter der genannten Creative Commons Lizenz steht und die betreffende Handlung nicht nach gesetzlichen Vorschriften erlaubt ist, ist für die oben aufgeführten Weiterverwendungen des Materials die Einwilligung des jeweiligen Rechteinhabers einzuholen.

Weitere Details zur Lizenz entnehmen Sie bitte der Lizenzinformation auf http://creativecommons.org/ licenses/by/4.0/deed.de. 